\section{NOVEL BIOLUMINESCENT BIOASSAYS FOR THE DISCOVERY AND DEVELOPMENT OF T CELL REDIRECTING CANCER THERAPIES}

Vanessa Ott*, Julia Gilden, Jamison Grailer, Michael Slater, Pete Stecha, Jim Hartnett, Dan Lazar, Frank Fan, Mei Cong, Zhijie Jey Cheng. Promega, Madison, USA

Background Two main approaches for $\mathrm{T}$ cell-based therapies involve molecular $\mathrm{T}$ cell redirection by $\mathrm{CD} 3$ bispecific molecules such as bispecific T-cell engagers (BiTE) and cellular $\mathrm{T}$ cell redirection by genetic modification of $\mathrm{T}$ cells with chimeric antigen receptors (CAR) or transgenic $\mathrm{T}$ cell receptors (TCR). BiTEs redirect the cytotoxic activity of endogenous polyclonal $\mathrm{T}$ cells by simultaneously engaging $\mathrm{CD} 3$ on $\mathrm{T}$ cells and tumor antigens on target cells. BiTE potency studies have relied on primary cells, which measure target cell killing through redirected $\mathrm{T}$ cell cytotoxicity (RTCC) or cytokine release. However, these primary cell-based assays suffer from high donor-to-donor variability, as well as lengthy and hard to implement protocols

Methods We have recently developed a new RTCC assay and cytokine immunoassays that are simple, sensitive and can quantitatively measure the potency of BiTEs and similar biologics. In this assay, preactivated cytotoxic $\mathrm{T}$ cells and target cells (both in cryopreserved thaw-and-use format) stably expressing a HaloTag-HiBiT fusion protein are co-incubated with a BiTE, which results in lysis of the target cells and subsequent release of the Halotag-HiBiT protein. These HiBiT proteins then bind to extracellular LgBiT provided in the detection reagent and form functional NanoLuc Luciferase to generate luminescence.

Results The assay is homogenous, highly sensitive, and has a robust assay window. Use of CAR-T has demonstrated promising results in treating leukemia, while the development of TCR-engineered $\mathrm{T}$ cells that can recognize intracellular tumor antigens, is still in early stages. To facilitate the screening and characterization of new transgenic TCRs, we used CRISPR/ Cas9 to develop two TCRaß-null reporter $\mathrm{T}$ cell lines, which are $\mathrm{CD} 4+$ or $\mathrm{CD} 8+$. Reintroduction of peptide-specific TCR $a$ and $\beta$ chains into TCRaß-null reporter $T$ cell lines results in peptide-dependent TCR activation and luciferase reporter expression. The select expression of CD4 or CD8 in the TCRaß-null reporter $\mathrm{T}$ cell lines can enable the development of transgenic TCRs for both MHCI- and MHCII-restricted tumor antigen targets.

Conclusions Together, these bioluminescent bioassays represent a new set of tools for the discovery and development of $\mathrm{T}$ cell-based immunotherapies.

http://dx.doi.org/10.1136/jitc-2020-SITC2020.0766

\section{INTERFERON GAMMA REDUCES CAR-T EXHAUSTION AND TOXICITY WITHOUT COMPROMISING THERAPEUTIC EFFICACY IN HEMATOLOGIC MALIGNANCIES}

Stefanie Bailey*, Sonika Vatsa, Amanda Bouffard, Rebecca Larson, Irene Scarfo, Michael Kann, Andrea Schmidts, Marcela Maus. Massachusetts General Hospital, Charlestown, MA, USA

Background Chimeric antigen receptor (CAR) T cell therapy has shown remarkable efficacy in hematologic malignancies, ultimately leading to its FDA approval for relapsed/refractory acute lymphoblastic leukemia and large cell lymphomas in
2017. Despite the success of CAR T cells in the clinic, toxicities such as cytokine release syndrome (CRS) can be severe. Attempts to mitigate these effects have primarily focused on the blockade of macrophage-derived cytokines, such as IL-6 and IL-1B. Herein, we show that the pharmaceutical blockade or genetic deletion of interferon gamma (IFNg, a CAR-Tderived cytokine that strongly correlates with CRS in the clinic, appears to be a viable target for the reduction of CART-associated toxicities.

Methods Pharmacologic (blocking antibody) and genetic (CRISPR/Cas9) approaches were used to block IFNg signaling and/or production by CAR $\mathrm{T}$ cells. In vitro CAR-T function and cytotoxicity was tested using ELISA, flow cytometry and short-/long-term killing assays prior to their assessment in vivo. NSG mice were injected with Nalm6 or JeKo-1 cancer cells prior to treatment with IFNg-modified CAR-T and tumor size and IFNg production were measured. To determine how the loss of IFNg might affect innate immune cells, CAR-T, macrophages and tumor cells were co-cultured and assessed by flow cytometry, immunofluorescence, Luminex and RNA sequencing.

Results IFNg could be blocked using an anti-IFNg antibody or CRISPR/Cas9 editing of the CAR T cells without affecting T cell activation, proliferation or cytokine production (IL-2, TNFa, GM-CSF). Successful blockade of the IFNg signaling pathway was confirmed by reduced phosphorylation of JAK1, JAK2 and STAT1, even in the presence of exogenous IFNg. Loss of IFNg did not reduce the cytotoxic potential or persistence of CAR-T against hematologic malignancies in vitro or in vivo. When cultured with macrophages and cancer cells, IFNg knockout (IFNgKO) CAR-T yielded decreased levels of IL-1B, IL-6, IL-13, MCP1 and CXCL10, indicating a reduction in macrophage activation induced by CAR-T in the absence of IFNg. Serum from tumor-bearing mice treated with IFNgKO CAR-T elicited lower activation of macrophages in vitro compared to those treated with IFNg-producing CAR-T cells. Furthermore, IFNgKO CAR $\mathrm{T}$ cells co-cultured with tumor cells and macrophages demonstrated less exhaustion as shown by reduced expression of PD1, Tim3 and Lag3 and increased IFNgKO CAR-T expansion.

Conclusions Collectively, these data suggest that IFNg is not required for the efficacy of CAR-T in hematologic malignancies and can potentially be targeted to reduce toxicity and enhance CAR-T efficacy and persistence in the clinic.

http://dx.doi.org/10.1136/jitc-2020-SITC2020.0767

\section{RE-DIRECTED T CELL THERAPY TO CONTROL INVASIVE ASPERGILLOSIS}

${ }^{1}$ Karishma Bavisi ${ }^{*},{ }^{1}$ Sebastian Wurster, ${ }^{1}$ Nathaniel Albert, ${ }^{1}$ Sattva Neelapu, ${ }^{1}$ Dimitrios P Kontoyiannis, ${ }^{2}$ Pappanaicken Kumaresan. 'University of Texas MD Anderson Cancer Center, Houston, TX, USA; ' ${ }^{2}$ University of Texas MD Anderson Cancer C, Sugar Land, TX, USA

Background Opportunistic invasive fungal infections (IFI) are a major threat to immunocompromised populations such as patients with acute myeloid leukemia (AML) and allogenic hematopoietic stem cell transplant (HSCT) recipients(1,2). Specifically, Aspergillus fumigatus (AF) is responsible for high morbidity and mortality in cancer patients. As antifungal therapy has limited efficacy in immunocompromised patients, we 